\title{
Buteyko Breathing Technique:The Golden Way for Controlling Asthma among Children
}

\author{
1Esraa Elwan Mohammed Hassan, 2 Fawzia Elsayed Abusaad, 3Boshra Attia Mohammed \\ 1Demonstrator in Pediatric Nursing Department, Faculty of Nursing, Mansoura University \\ 2Professor of Pediatric Nursing, Faculty of Nursing, Mansoura University, 3Assistant Professor of \\ Pediatric Nursing, Faculty of Nursing, Mansoura University \\ E-mail:1esraa_elwan@mans.edu.eg\&1esraaelwan1992@gmail.com \\ 2 fawziaabusaad2013@gmail.com\&3toboshra@yahoo.com \\ Corresponding author: 1 Esraa Elwan Mohammed Hassan
}

\begin{abstract}
Asthma is one of the chronic respiratory disorder affecting children of all age group .It is characterized by long-term airway inflammation with respiratory symptoms such as dyspnea, wheezes, shortness of breath and episodes of coughing of variable severity. The possible effects of asthma and other chronic respiratory problems to children are often attributed to restrictions in activity and its dangerous consequences. Despite there is a better awareness of the causes that cause asthma and new pharmacological measures are available for the treatment of asthma, the goal is to ensure that as many children with asthma worldwide have access to cost-effective management methods to minimize morbidity and mortality as possible.This can be achieved by combination of medical therapy and complementary alternative medicine techniques. One such method is Buteyko breathing technique (BBT). This technique is a distinctive breathing treatment that uses breathing control and breath-holding techniques to treat a wide variety of health problems that are suspected to be due to hyperventilation as bronchial asthma. This article review describes the process of $B B T$, the physiology behind it, and discusses the available evidence for its efficacy.
\end{abstract}

Keywords:Buteyko Breathing Technique, Childhood Asthma, Controlling Asthma, Effectiveness. 


\section{Introduction}

Asthma is one of the most prevalent chronic disorders affecting bronchial trees that triggered by hyperresponsiveness of the airways. It was described as the narrowing of the airways in response to a variety of stimuli (World Health Organization (WHO), 2020).It causes intermittent breathing difficulties that are marked by completely or partially reversible airflow limitation, and may spontaneously improve or subside only after specific treatment.This symptom appears especially at night and early morning, which may be life threatening if left untreated, but can be reversible with proper care (El-Gilany, El Desoky, ElHawary, \& Farrag, 2018). All over the world, asthma is one of the prevalent non-communicable diseases (NCDs) with large variations in prevalence and severity. For the future, it may remain a challenge and still receive insufficient support and treatment.There are around 300 million people diagnosed with asthma. In the last 40 years, the incidence of allergy-related asthma has grown. By 2025, the number of individuals with asthma will rise by more than 100 million (Nunes, Pereira, \& Morais-Almeida, 2017;WHO, 2020). Extreme and uncontrolled asthma can endanger life. Many medications are used for the treatment of children with asthma. However, they have not been shown to adequately improve the quality of life (QoL). Moreover, they also have severe adverse effects that hinder their long-term use (Global Initiative for Asthma, 2019). It is important to have quick and economical managementto effectively monitor asthma symptoms and reduce the incidence of acute exacerbation, which prevents its harsh consequences from restricting the growth and development of the child (Mohamed, Elderiny, \& Ibrahim, 2017;Kalaci, et.al.,2019). This can be done by combination of pharmacological therapy and other complementary alternative medicine (CAM), one of these alternative therapiesis the Buteykobreathing technique (BBT). It was developed by Dr. Konstantin Buteyko in Russia in 1952, which believed that hyperventilation induces asthma. Buteyko approach seeks to educate asthmatic patients to decrease airflow by teaching them the best way to hold their breath at the functional residual capacity (Mohamed, et.al., 2017). Global interest in the BBT has been picking up in recent years, but further studies are possibly also needed to be approved by the public and in particular by the healthcare team (Ward, \& Baptist, 2016).

\section{Literature Searching Strategy}

The authors searched electronic medical and health care databases, including "Google Scholar", "Cochrane library", "Science Direct","PubMed", "Scopus", "DOAJ", "ERIC", and "EBSCOhost", to find appropriate literature on this subject.As keywords, the following search phrases were used: "Buteyko breathing technique, "Buteyko breathing exercise," "complementary alternative therapy," "asthma," "childhood asthma," "asthma physical therapy," "breathe holding".

\section{Background about Asthma}

Asthma is a long-lasting illness that varies and fluctuates over time, happens frequently in early childhood and is the dominant constant illness in children (WHO, 2020). There is no fully accepted definition of asthma to date, which encompasses children from birth to puberty. It can be operationally 
described as a bronchial tree obstructive disease due to airway smooth muscle contraction, increase secretion, and swelling or inflammation of the airway (Ahmed, \& Turner, 2019). Asthma affects all age classes, but mostly begins in infancy. It is a condition characterized by repeated breathlessness and wheezing attacks, which vary in severity and duration from person to person. Moreover, for the same person, it can vary from hour to hour and day to day (WHO, 2020). Childhood asthma make the child, their families and community under a substantial burden. It is associated with poor lung function development, this impairment increase the risk of chronic obstructive pulmonary disease and affect the respiratory health in adult life (El-Gilany, et.al., 2018).A sharp rise in asthma incidence was observed in a number of developing countries during the second half of the twentieth century, especially after the 1960 (Dharmage, Perret, \& Custovic, 2019). About 300 million people globally affected by asthma which it is a persistent condition for both children and adults, and it is likely that another 100 million will be affected by 2025 (Nunes, et.al., 2017). The asthma burden is considerable. In developing countries, over $80 \%$ of asthma-related deaths occur (WHO, 2020). Asthma, particularly among children from poor families, is a common health problem in Egypt and is possibly underdiagnosed and undertreated (El-Gilany, et.al., 2018).In Delta region the childhood asthma overall prevalence is 13.4 percent. In boys, it is substantially higher than girls (15.4 percent vs.11.3 percent) (Meatty, El-Desoky, El-Domyaty, El-Gilany, \& Nasef, 2018). Ventilation restriction in asthma is frequent and influenced by a variety of airway changes
(Bush,2019). The dominant physiological event in asthma contributing to clinical signs and subsequent disruption of airflow is bronchoconstriction. In acute asthma exacerbations, this bronchoconstriction arise in response to exposure to a number of stimuli, including allergens or irritants, that rapidly narrows the airways (Sanak, 2016;Fehrenbach, Wagner, \& Wegmann, 2017). Other variables further limit airflow as the disease becomes more persistent and constriction becomes more progressive. Which include edema, inflammation, increase mucus secretion and the formation of phlegm, as well as structural changes, including smooth muscle airway hypertrophy and hyperplasia. These latter alterations may not respond to standard therapy (Inyor, \& Perez,2019). Asthma is a complex condition that includes biochemical, autonomic nervous system, immunological, viral, endocrine, and psychosocial factors of different degrees in different individuals. Therefore, the causes of childhood asthma are not well known (Meatty, et.al., 2018). The risk factors for asthma include genetic patterns, family history, certain forms of airway infections at a very early age, susceptibility to environmental triggers such as tobacco smoking, dust mite, pet dander, pollen, physical activity, variations in temperature, food allergy, obesity, and emotions. Sometimes with no visible triggers, asthma attacks arise (Bhattacharjee, et.al., 2018). Recurrent wheezing, coughing, chest tightness, and dyspnea are signs and symptoms of asthma, which becoming more common at nighttime and early morning, that often diminishing QoL (Thomson, 2015). Diagnosing a child as having asthma is only the first step in reducing symptoms, physical disabilities, 
QoL deficiency, and the possibility of disease-related adverse effects (AlMoamary, et.al., 2019). The diagnosis of asthma is based on history, physical examination, lung function examinations, and other differential diagnosis assessments (Horak, et.al., 2016). There is no cure for asthma, but with the proper diagnosis, care and an asthma control plan, the symptoms can be controlled (Tesse, Borrelli, Mongelli, Mastrorilli, \& Cardinale, 2018). Controlling of asthma concentrates on two areas: first reducing impairment-the recurrence and severity of a child's immediate or recent signs and functional limitations; and the second area is to reducing risk-the probability of potential asthma attacks, gradual reduction in lung capacity (or decreased lung development for children), or side effects of medication (Al-Moamary, et.al., 2019). Childhood asthma poses special challenges. The child's age, the fluctuating nature of asthma incidence, clinical presentation instability, exacerbations, comorbidities, socio-economic and psychosocial influences, and environmental factors can all affect the activity of the disease and thus, strategies for monitoring (Tesse, et.al., 2018). Children with mild to serious exacerbations can be treated effectively with bronchodilators and oral steroids in the emergency department, as outpatients. Hospitalization to the pediatric intensive care unit (PICU) should be prompt in extreme exacerbations, while life-threatening risks of asthma could occur. Children with a sudden exacerbation of asthma needs immediate care and assessment. The primary principles of therapy are to alleviate blocking of airflow, enhance respiratory work and improve oxygenation and perfusion of tissue (Arakawa, et.al., 2017).Asthma pharmacological therapy involves; reliever medicines- that enable symptom relief within a few minutes, during asthma worsening or exacerbations; and control medicines-that are used to maintain asthma care (Ellwood, et.al. 2017). Beta-adrenergic agonist has long been the cornerstone of asthma medicine, in which the key of acute asthma treatment is inhaled short-acting beta-2 agonist (SABA) therapy. Systemic steroids as an anti-inflammatory agent are also important in the treatment of asthma. It is essential in managing persistent inflammation and stabilizing the exacerbation of acute asthma. Intracellular magnesium sulfate, that is essential for airway smooth muscle relaxation and mast cell release of histamine, was found to be weak in children with acute asthma exacerbation. In children with less than 50 percent peak expiratory flow rate (PEFR) who have not had a substantial reaction to inhaled bronchodilators, magnesium sulfate should be considered. (Hunter, Vaddi, Krvavac, Regunath, \& Guntur, 2019). In all cases of asthma exacerbation, hypoxemia should be treated with supplementary oxygen with a $\mathrm{SpO} 2$ target of 93-95 percent and frequent reassessments are required.In acute asthma exacerbations, recommendations advocate against routine use of antibiotics. In those without signs and symptoms of inflammation, the antibiotic use not recommended in the recent studies (Stefan, et.al., 2020).

Complementary Alternative Therapy for Asthma

However there is a deeper recognition of the causes of asthma and new public health and pharmacological approaches are available to decrease the occurrence of asthma, the purpose is to 
ensure that as many individuals with asthma globally as possible have access to cost-effective measures to minimize morbidity and mortality (Ellwood, et.al., 2017). However these new developments in asthma pharmacological intervention, the global public concern is in physical therapy and Complementary Alternative Medicine (CAM) for asthma (Quirt, Hildebrand, Mazza, Noya, \& Kim, 2018). The irregular breathing patterns can be stabilized by additional CAM. The use of acupuncture, air purifiers or ionizers, homeopathy, yoga, massage, breathing exercises, calming treatments, herbal medicine, salt therapy, dietary therapies, and traditional Chinese medicine has grown substantially over the last 10 years in Europe, the United States and Australia (Ward, \& Baptist, 2016). One such technique gaining popularity is the Buteyko Breathing Technique (BBT).There is inadequate evidence to support the recommendation of asthma therapy by using BBT guideline, especially in developed countries. In addition, more study in this area is also needed to raise understanding of BBT in order to gain better control over bronchial asthma(Mohamed, et.al., 2017; Hunter, et.al., 2019).

Background and Theoretical Basis about Buteyko Breathing Technique

It was originally discovered by Russian clinical physician Dr. Konstantin Pavlovich Buteyko in the 1952. In the early 1990s, the Buteyko process first appeared in Australia and the UK from Russia. Early on, this technique was popular with adults and children suffering from asthma. The Buteyko procedure has been shown to be successful in improving a variety of breathing difficulties over the past few years. (Mohamed, et.al., 2017). The hypothesis of the technique was that hyperventilation made asthma worse. A common, important, and generally unrecognized destabilizer of physiological processes and psychological states was thought to be the persistent hidden hyperventilation (Hunter, et.al., 2019).

In Buteyko's opinion, the body evolved a series of defense mechanisms to preserve $\mathrm{Co} 2$, including constriction of airways and blood vessels, and triggering diseases such as asthma and hypertension, since carbon dioxide (Co2) was so essential. Carbon dioxide $\mathrm{Co} 2$ is a pulmonary bronchodilator. "hypocapnia" with low $\mathrm{Co} 2$ has exacerbated many medical complications and has created as many as 150 symptoms and disorders (Courtney, 2014).In asthma, there are four main consequences of excess breathing; lowering levels of $\mathrm{Co} 2$. This low level may cause the smooth muscle around the bronchioles to spasm. This resulting in chest tightness and difficulties exhaling and Oxygen is expelled from the blood more slowly creating breathlessness (Hassan, Riad, \& Ahmed, 2012).

\section{Buteyko Breathing Technique}

$$
\text { The Buteyko breathing }
$$

technique's methods are unusual in which asthmatic patients are needed to breathe less than more and not breathing or holding the breath is considered important as breathing. The main component of the BBT is to minimize hyperventilation, known as 'slow breathing' and 'reduced breathing' by periods of regulated breathing reduction, combined with periods of breath holding, known as 'control pauses' and 'extended pauses'(McKeown, 2010). The use of the diaphragm for breathing is often recommended and the use of accessory muscles for breathing is discouraged. 
These techniques are very similar to those that respiratory physiotherapists routinely use for patients with symptoms of hyperventilation (Courtney, 2014).

The procedure in Buteyko is often supplemented by physical activities to improve the build-up of Co2. With a minor rise in $\mathrm{Co} 2$ to normal, there are several beneficial effects in the body, including relaxation of smooth muscle, increasing oxygenation, switching on the relaxing nervous system and increasing the body's production of nitric oxide(McKeown, 2010). Bronchi relaxing and narrower airways, bronchioles, can improve circulation and significantly reduce asthma-related airway spasms. In the Buteyko method, a tip for nasal breathing over oral breathing is also used . The nose warm up, filter and humidify the inspired air. In addition, it releases nitric oxide, which is an effective asthma bronchodilator. Patients with Buteyko are advised to breathe through the nose during the day and try to tap the mouth at night to facilitate nasal breathing. The Buteyko technique also proposes lifestyle changes beyond breathing, including diet, allergy avoidance, and stress control (Rahi, Jaber, \& Abd-Ali, 2019).

\section{Benefits of Buteyko Breathing} Technique

A variety of clinical trials have demonstrated that it is an effective treatment for asthma, but there is little evidence for the $\mathrm{Co} 2$ hypothesis behind the Buteyko process (Hunter, et.al., 2019). In addition to $\mathrm{CO} 2$ theory, there are also other potential explanations why the methods of breathing used by the Buteyko system work. These potential explanations include improvements in the perception of symptoms and increased sense of balance, enhanced breathing physiology, beneficial effects of low-volume breathing, altered levels of nitric oxide, and resetting respiratory function by breathing strategies (Rahi, et.al., 2019).

Buteyko breathing rules

When practicing Buteyko breathing, the first thing to be remember is inhalation in a very controlled and shallow manner. There must be a gentle in and out breathing rhythm. The mouth should be maintained closed throughout all times and the nose used only for breathing. If the child has a blocked nose, it may be difficult, but once the breathing technique starts, it should eventually clear up (Bruton, \& Lewith, 2005 ;McKeown, 2010). The diaphragm must be inflates and deflates in a controlled rhythm and that the chest stays still. The breathing must be in a very shallow manner. The four cardinal rules of the Buteyko method are as follows: holding the mouth closed, keeping the back straight, breathing quietly and silently, and eating only if hungry (Hassan, et.al., 2012).

\section{Research Studies on the Buteyko} Method

A number of clinical trials indicate that it may be a successful therapy for asthma, but the $\mathrm{Co} 2$ hypothesis behind the Buteyko method is little supported (Hunter, et.al., 2019). Early on, the procedure was extended to adults and later to children with asthma. In recent years, it has been successful in improving a range of respiratory difficulties (Mohamed, et.al., 2017). As presented byBruton, \& Lewith, (2005) how conducted an article review about "Buteyko breathing technique for asthma" and found that the suggestion so far, and there was inadequate evidence to approve that the theory concerning to carbon dioxide levels is the mechanism behind any effect that BBT may exert. 
The study concluded that further analysis is important to decide whether BBT is successful and, if so, how it operates. Anotherarticle review conducted by Courtney, (2008)about the "strengths, weaknesses, and possibilities of the Buteyko breathing method" and found thatthe BBT is still not widely known in the society by public and medical staff. This may be because the carbon dioxide philosophy that underpins this approach has not been reinforced by research.

After that, in the last ten years many researches and articles about Buteyko are published. A systemic review article was conducted by Burgess, et.al., (2011) about "the effectiveness of breathing retraining in asthma management".It was found thathowever, very few well-designed trials with sufficient strength and duration of follow-up were found to allow definitive conclusions to be drawn, it was proposed that it would be appropriate for physicians to provide trained asthma patient care and conduct certain procedures under the guidance of a qualified practitioner. This article was followed by arandomized controlled trialamong asthmatic adult patients that conducted by Prem, Sahoo, \& Adhikari, (2013). It was"compared the effects of Buteyko and pranayama breathing techniques on quality of life in patients with asthma" and found that the Buteyko group exhibited greater patterns of progress in quality of life and management of asthma than the pranayama breathing exercise group. Another study conducted by Villareal, et.al.,(2014) to assess the "Effect of Buteyko method on asthma control and quality of life of Filipino adults with bronchial asthma". It was corresponding to the previous results and supported that when used as acombined therapy in adults with bronchial asthma, the Buteyko approach has an important beneficial effect on asthma management and quality of life. Recently two clinical trial among adult was established. The first one conducted byJinsabin, (2018) and designed to compare the "effectiveness of Buteyko breathing technique and active cycle of breathing technique in the management of dyspnea among asthmatic patients'.It revealed that BBT was seen to be efficient in lowering dyspnea and enhancing the rhythm of breathing as opposed to the active breathing cycle. The second trial conducted in Airlangga by Udayani, Amin, \& Makhfudli, (2019) and proposed to study the "the effect of combination of Buteyko breathing technique and walking exercise on forced peak expiratory flow in adult asthmatic patients". Its results found that breathing techniques and physical activity would improve the PEFR levels by a mixture of Buteyko breathing strategies and walking exercise that can decrease the symptoms of asthma. In addition, it concluded that this technique should be used as an alternate option for asthmatic patients to support pharmacological treatment. Among children, a study conducted by Lina, et.al., (2013) about "effectiveness of Buteyko method in asthma control and quality of life of school-age children"also supported the Buteyko technique.It noted that the use of the Buteyko technique as an alternative to traditional asthma treatment within three to four weeks tends to enhance schoolage children's asthma management and quality of life. Anothersingle blind randomized controlled trial of Buteykotechnique for children withasthma that assessed 32 children aged seven to twelve years old with asthma was conducted by Mendonca, 
et.al.,(2017). It concluded that the Buteykotechnique has been useful in improving some parameters of lung function and symptoms. Both the Buteyko strategy and asthma education have strengthened children's QoL. In Egypt, this technique was applied through five studies, four among adult patients and only one among children. The first one conducted in Cairoand Ain ShamsUniversitiesby Hassan, et.al., (2012) that was applied on 40 patients with bronchial asthma their age ranged between 30 and 50 years recruited from 23rd of July Chest Hospital. After the applied program that continued for six weeks, the results of the study revealed a large reduction in everyday symptoms of asthma, a major increase in PEFR, and a control pause test in the study group over control one. This study followed by another one in the same university but it was applied among 54 children. The study conducted by Elnaggar, \& Shendy, (2016) and aimed to "compare the effects of the active cycleof breathing technique (ACBT), Buteyko breathing technique (BBT), and thoracic lymphatic pump technique (TLPT) on the total serum immunoglobulin (Ig) E, ventilator function, and asthma perception in children with bronchial asthma". The result revealed that, BBT and TLPT are more effective in the care of children with bronchial asthma compared with ACBT. Moreover, Buteyko's breathing is theoretically more valuable.In chest medicine ward and clinic at Ain Shams University Hospital another comparative study conducted by Mohamed, Elderiny, \& Ibrahim, (2017) to assess the "The effect of Buteyko breathing technique among patients with bronchial asthma". It was concluded that practicing BBT has been shown to have a beneficial impact on reducing the incidence of asthma symptoms and improving the ability to control asthmatic patients. This study followed by another one among adult conducted in chest disease department at Port-said El- Masah ElBahry Hospital by Mohamed, ELmetwaly, \& Ibrahim, (2018). The efficacy of Buteyko breathing exercise over normal care in asthmatic patients was confirmed by this study outcome, as there was a significant statistical increase in the regulation of daily asthma, seriousness of asthma, and pulmonary function in patients for one month after Buteyko breathing technique was applied over pre-applied.

In Mansoura University, only one study conducted in faculty of nursing by Abouelala, Sherif, Elshamy, \& Shalabi, (2017). It was aimed to assess the effect of Buteyko breathing exercise on the asthma control and quality of life among 90 adult patient divided to experimental and control group from outpatient of chest disease department in Mansoura university hospital over a period of twelve months. The study result showed a major decline in rescue drug, seriousness of asthma and increase in Buteyko group asthma control and quality of life than control one. It also suggested the early introduction of BBT to asthmatic patients and the establishment of a BBT clinic with trained Buteyko practitioners in the chest department.

\section{Conclusion}

This article review describes the process of BBT, the physiology behind it, and discusses the available evidence for its efficacy. Currently, there is actually no asthma cure, so treatments aims to control or relieve symptoms. Buteyko breathing technique is a complementary therapy that have been discovered to serve this aim. The 
Classical Buteyko theory propose that the mechanism behind its effect is the changes in $\mathrm{CO} 2$ level.Despite the growing body of knowledge about this technique, guarded support by health and government officials, and the excitement of people who have been supported,more studiesespecially among childrenare required to confirm or deny the opinion that modifying breathing habits will potentially have a major influence.

\section{References}

- Abouelala, F. M., Sherif, W. I., Elshamy, K. F., \& Shalabi N. M., (2017). Impact of Buteyko Breathing Technique on the Control of Bronchial Asthma and Quality Of Life among Asthmatic Patients at Mansoura University Hospital. Unpublished Doctoral thesis.

- $\quad$ Ahmed, H., \& Turner, S. (2019). Severe asthma in children-a review of definitions, epidemiology, and treatment options in 2019. Pediatric pulmonology, 54(6), 778-787

- Al-Moamary, M. S., Alhaider, S. A., Alangari, A. A., Al Ghobain, M. O., Zeitouni, M. O., Idrees, M.M., ... \& Al-Hajjaj, M. S. (2019). The Saudi Initiative for Asthma-2019 Update: Guidelines for the diagnosis and management of asthma in adults and children. Annals of thoracic medicine, 14(1), 3.

- Arakawa, H., Hamasaki, Y., Kohno, Y., Ebisawa, M., Kondo, N., Nishima, S., ... \& Morikawa, A. (2017). Japanese guidelines for childhood asthma 2017. Allergology International, 66(2), 190-204.

- Bhattacharjee, S., Haldar, P., Maity, S. G., Debnath, S.,
Moitra, S., Saha, S., ... \& Moitra, S. (2018). Prevalence and Risk Factors of Asthma and AllergyRelated Diseases among Adolescents (PERFORMANCE) study: rationale and methods. ERJ open research, 4(2), 00034-2018.

- Bruton, A., \& Lewith, G. T. (2005). The Buteyko breathing technique for asthma: a review. Complementary therapies in medicine, 13(1), 41-46.

Bush, A. (2019). Pathophysiological mechanisms of asthma. Frontiers in pediatrics, 7.

- Burgess, J., Ekanayake, B., Lowe, A., Dunt, D., Thien, F., \& Dharmage, S. C. (2011). Systematic review of the effectiveness of breathing retraining in asthma management. Expert review of respiratory medicine, 5(6), 789807.

- Courtney, R. (2008). Strengths, weaknesses, and possibilities of the Buteyko breathing method. Biofeedback, 36(2), 5963. Available at https://buteykoclinic.com/docs/stre ngths\%2Bweaknesses\%2Band\%2 Bpossibilities 1402584111.pdf.

- Courtney, R. (2014). Buteyko breathing method. Recognizing and Treating Breathing Disorders E-Book, 241.

- Dharmage, S. C., Perret, J., \& Custovic, A. (2019). Epidemiology of asthma in children and adults. Frontiers in pediatrics, 7, 246. Avilable at https://www.frontiersin.org/article s/10.3389/fped.2019.00246/full

- El-Gilany, A. H., El Desoky, T., El-Hawary, A. K., \& Farrag, M. (2018). Quality of life of children 
with bronchial asthma and their caregivers: A hospital-based study. Progress in Medical Sciences, 2(1), 1. Available at http://www.promedsci.org/fulltext/ 179-1524127729.pdf?1591950601

- Ellwood, P., Asher, M. I., Billo, N. E., Bissell, K., Chiang, C. Y., Ellwood, E. M., ... \& Pearce, N. E. (2017). The Global Asthma Network rationale and methods for Phase I global surveillance: prevalence, severity, management and risk factors. European respiratory journal, 49(1), 1601605. Available https://erj.ersjournals.com/content/ erj/49/1/1601605.full.pdf

- Elnaggar, R. K., \& Shendy, M. A. (2016). Efficacy of noninvasive respiratory techniques in the treatment of children with bronchial asthma: a randomized controlled trial. Bulletin of Faculty of Physical Therapy, 21(1), 1-10.

- Fehrenbach, H., Wagner, C., \& Wegmann, M. (2017). Airway remodeling in asthma: what really matters. Cell and tissue research, 367(3), 551-569. Available

at
https://link.springer.com/article/10 $.1007 / \mathrm{s} 00441-016-2566-8$

- Global Initiative for Asthma (2019), global strategy for asthma management and prevention, available

https://ginasthma.org/wpcontent/uploads/2019/06/GINA2019-main-report-June-2019wms.pdf

- Hassan, Z. M., Riad, N. M., \& Ahmed, F. H. (2012). Effect of Buteyko breathing technique on patients with bronchial asthma. Egyptian Journal of
Chest Diseases and

Tuberculosis, 61(4), 235-241.

- Horak, F., Doberer, D., Eber, E., Horak, E., Pohl, W., Riedler, J., ... \& Studnicka, M. (2016). Diagnosis and management of asthma-Statement on the 2015 GINA Guidelines. Wiener Klinische Wochenschrift, 128(1516), 541-554.

- Hunter, M. P., Vaddi, S., Krvavac, A., Regunath, H., \& Guntur, V. P. (2019). Inpatient Management of Bronchial Asthma for the Hospitalist-A Concise Review. Am J Hosp Med, 3(4).

- Inyor, B., \& Perez, L. C. (2019). Pathophysiology Of Asthma. In StatPearls [Internet]. StatPearls Publishing.

- Jinsabin, M. L. (2018). $A$ Comparative study on Effectiveness of Buteyko Breathing Technique and Active Cycle of Breathing Technique in the Management of Dyspnoea among Asthma Patients (Doctoral dissertation, Cherraan's College of Physiotherapy, Coimbatore).

- Kalaci, O., Giangioppo, S., Leung, G., Radhakrishnan, A., Fleischer, E., Lyttle, B., ... \& Radhakrishnan, D. (2019). Complementary and alternative medicine use in children with asthma. Complementary Therapies in Clinical Practice, 35, 272-277.

- Lina, R. C., Leysa, M. D. V., Libozada, Z. D., Lirio, M. F. I., Liwag, A. A., Ramos, G. D., \& Natividad, M. M. (2013). Effectiveness of Buteyko method in asthma control and quality of life of school-age children.

- McKeown, P. (2010). Buteyko Meets Dr. Mew. Asthma Care 
Buteyko Clinic. Available at https://books.google.com.sa/books ?hl=en\&lr=\&id=LAA 1DZD2BY C\&oi $=$ fnd\&pg $=$ PA1\&dq $=$ Buteyko + Meets + Dr. + Mew\&ots $=$ T0pFhsz Gho\&sig=cxuiIvZX1rDNU61uwao zmeaJZWs\&redir esc $=\mathrm{y} \# \mathrm{v}=$ onepa ge\&q=Buteyko $\% 20$ Meets $\% 20$ Dr. $\% 20 \mathrm{Mew} \& \mathrm{f}=$ false

- Meatty, E. M., El-Desoky, T., ElDomyaty, H., El-Gilany, A., \& Nasef, N. (2018). Prevalence of childhood bronchial asthma and its associated factors: A communitybased study in Egypt. Progress Med Sci, 2(2), 14-20.

- Mendonca, K. M., Freitas, D. A., Macedo, T. M.,Silva, A. C., Amaral, C. T., Santino, T. A., \& McKeown, P. (2017). Buteyko Method For Children With Asthma: A Randomized Controlled Trial. In A66. THE MANY FACES OF ASTHMA IN CHILDHOOD (pp. A2203A2203). American Thoracic Society.

- Mohamed, E. M. H., ELmetwaly, A. A. M., \& Ibrahim, A. M. (2018). Buteyko Breathing Technique: A Golden Cure for Asthma. American Journal of Nursing, 6(6), 616-624.

- Mohamed, Y., Elderiny, S., \& Ibrahim, L. (2017). The effect of Buteyko breathing technique among patients with bronchial asthma: Comparative study. International Journal of Midwifery and Nursing Practice 2019; 2(2): 01-10. available at https://www.researchgate.net/profi le/Yosreah_Mohamed2/publicatio $\mathrm{n} / 335957160$ The Effect of Bute yko Breathing Technique among Patients with Bronchial Asthma
Comparative Study/links/5d8570 6a458515cbd1a3f582/.pdf

- Nunes, C., Pereira, A. M., \& Morais-Almeida, M. (2017). Asthma costs and social impact. Asthma research and practice, 3(1), 1.

- Prem, V., Sahoo, R. C., \& Adhikari, P. (2013). Comparison of the effects of Buteyko and pranayama breathing techniques on quality of life in patients with asthma-a randomized controlled trial. Clinical rehabilitation, 27(2), 133-141.

- Quirt, J., Hildebrand, K. J., Mazza, J., Noya, F., \& Kim, H. (2018). Asthma. Allergy, asthma, and clinical immunology : official journal of the Canadian Society of Allergy and Clinical Immunology, 14(Suppl 2), 50. https://doi.org/10.1186/s13223018-0279-0

- Rahi, A. A., Jaber, A. M., \& Abd-Ali, D. K. (2019). Effect of Buteyko Method on Lungs Functions among Asthmatic Patients in Al-Najaf City. kufa Journal for Nursing sciences, 9(2), 1-7. Available at https://www.iasj.net/iasj?func=full text\&aId=174626

- Sanak, M. (2016). Eicosanoid mediators in the airway inflammation of asthmatic patients: what is new?. Allergy, asthma \& immunology research, 8(6), 481-490.

- Stefan, M. S., Spitzer, K. A., Zulfiqar, S., Heineman, B. D., Hogan, T. P., Westafer, L. M., ... \& Lindenauer, P. K. (2020). Uncertainty as a critical determinant of antibiotic prescribing in patients with an 
asthma exacerbation: a qualitative study. Journal of Asthma, 1-16.

- $\quad$ Tesse, R., Borrelli, G., Mongelli, G., Mastrorilli, V., \& Cardinale, F. (2018). Treating pediatric asthma according guidelines. Frontiers in pediatrics, 6, 234.

- Thomson, N. C. (2015). Recent advances in the treatment and management of asthma. Prescriber, 26(18), 17-24.

- Udayani, W., Amin, M., \& Makhfudli, M. (2019). The Effect of Combination of Buteyko Breathing Technique and Walking Exercise on Forced Peak Expiratory Flow In Adult Asthmatic Patients. Jurnal Keperawatan Padjadjaran, 7(2).
- Villareal, G. M. C., Villazor, B. P. U., Villegas, A. M., Visaya, P. S. N., Vista, M. E., \& Tan, C. B. (2014). Effect of Buteyko Method on Asthma Control and Quality of Life of Filipino Adults with Bronchial Asthma. The Journal of Macro Trends in Health and Medicine, 1, 44-60.

- Ward, C. E., \& Baptist, A. P. (2016). Characteristics of Complementary and Alternative Medicine (CAM) use among older adults with asthma. Journal of Asthma, 53(5), 546-552.

- World Health Organization, Asthma, (2020). Available at https://www.who.int/newsroom/q-a-detail/asthma 